\title{
ZIF-8 and polyaniline modified coconut gel to fabricate composite aerogel for efficient removal of tetracycline
}

\author{
Qun Liu ${ }^{1,2}$, Fanming Zeng ${ }^{1,3^{*}}$, Xiao Li ${ }^{1,3}$, Zhongmin $\mathrm{Su}^{1,3}$ \\ ${ }^{1}$ School of Materials Science and Engineering, Changchun University of Science and Technology, Changchun 130022, China \\ ${ }^{2}$ Department of Light Chemical Engineering, Jilin Institute of Chemical Technology, Jilin 132022, China \\ ${ }^{3}$ Jilin Provincial Science and Technology Innovation Center of Optical Materials and Chemistry, Changchun, 130022, China
}

\begin{abstract}
To overcome the limitations of large-scale applications for MOFs in the powder form, herein, we proposed a strategy of in-situ growth ZIF-8 onto polyaniline (PANI) modified coconut hydrogel (CCH). Firstly, PANI played the role of metal chelated layers, which were coated on $\mathrm{CCH}$ by in-situ polymerization. Then, ZIF-8 nanocrystals were in-situ growth on the surface of the PANI coated CCH to synthesise the composite adsorbent ZIF-8/PANI/CCH. Finally, after vacuum freeze-dried, a white and well structured ZIF8/PANI/CCA(ZIF-8/polyaniline/coconut aerogel) was obtained. The loading mass ratios of ZIF-8 on CCA and PANI/CCA were $11.3 \%$ and $37.5 \%$, respectively, which indicates that PANI as interface layers can effectively promote the in-situ growth of ZIF-8. The obtained composite adsorbent (ZIF-8/PANI/CCA) was applied for the adsorption of tetracycline (TC), and the removal efficiency reaches over $91.6 \%$. This strategy may provide an effective and versatile pathway to increase MOF loading mass on natural polysaccharide aerogel and sequentially branch out their applications in pollutant treatment fields.
\end{abstract}

\section{Introduction}

Metal organic frameworks (MOFs), as a new class of porous materials, have shown great application potential in the field of wastewater treatment[1-2]. However, the nanopowder properties of MOFs greatly limit their practical applications[3-4]. A groundbreaking study has recently been reported to anchor MOFs to the surface of the layered structure of polysaccharide aerogels by insitu synthesis, while maintaining their efficient adsorption capacity[5-7]. However, the loading mass and robustness of MOF is limited by the low activity of hydroxyl groups on cellulose aerogels. The numerous quinone nitrogen atoms in Polyaniline (PANI) layers can coordinate with metal ions and create rich nucleation sites that are convenient to the growth of the MOF layers[8].

Herein, we report a method to fabricate ZIF8/PANI/coconut aerogel (ZIF-8/PANI/CCA) composites via in-situ polymerization and in-situ growth. Moreover, the ZIF-8/PANI/CCA was employed as adsorbent for the removal of antibiotic tetracycline through batch adsorption. This strategy may provide an effective and versatile pathway to increase MOF loading mass on aerogel and sequentially branch out their applications in pollutant treatment fields.

\section{Experimental}

\subsection{Materials and Characterization}

Coconut hydrogel was procured from supermarket. 2methylimidazole (98\%), Zinc nitrate $\left(\mathrm{Zn}\left(\mathrm{NO}_{3}\right)_{2} \cdot 6 \mathrm{H}_{2} \mathrm{O}\right.$, $99 \%)$ were acquired from Aladdin. Aniline (99.5\%) and Tetracycline (97.5\%) were purchased from Macklin. All other solvents were used without further purification. Powder X-ray diffraction (PXRD) spectra were measured ranging from $5-50^{\circ}$ on a Siemens D5005 diffractometer.

\subsection{Preparation of the MOF composite aerogel materials}

\subsubsection{Preparation of PANI/CCA by in-situ polymerization}

$\mathrm{CCH}$ (Coconut hydrogel) was immersed into $40 \mathrm{~mL} \mathrm{HCl}$ solution $(0.5 \mathrm{M})$ containing $912 \mu \mathrm{L}$ aniline and $10 \mathrm{~mL}$ $\mathrm{H}_{2} \mathrm{O}_{2} 30$ (wt, \%). Then in-situ polymerization of aniline to PANI was performed at room temperature for $24 \mathrm{~h}$. The prepared PANI/CCA was rinsed with $\mathrm{HCl}$ solution $(0.5 \mathrm{M})$ and deionized water. The protonated polyaniline layers were then converted to emeraldine base by treated with excess amount of ammonium hydroxide $(0.1 \mathrm{M})$, and then vacuum freeze-dried for $24 \mathrm{~h}$.

\footnotetext{
* Corresponding author: zengfm@cust.edu.cn
} 


\subsubsection{Preparation of ZIF-8/PANI/CCA and ZIF- 8/CCA by in-situ growth}

$3.39 \mathrm{~g}$ 2-methylimidazole was dissolved in $125 \mathrm{~mL}$ methanol to obtain solution A. $3.08 \mathrm{~g} \mathrm{Zn}\left(\mathrm{NO}_{3}\right)_{2} \cdot 6 \mathrm{H}_{2} \mathrm{O}$ was dissolved in $30 \mathrm{ml}$ methanol to obtain solution B. The hydrogel materials were added to solution $\mathrm{B}$ and stirred for $2 \mathrm{~h}$. Solution B was added to solution A under stirring. Stirring was continued for $4 \mathrm{~h}$ and allowed to stand for $12 \mathrm{~h}$. Take out the composite material and wash with methanol 3 times, then freeze-dry in vacuum for 24h. The pure ZIF-8 was obtained according to the above synthesis process without added gel materials.

The mass loading ratio of ZIF-8 on CCA and PANI/CCA were calculated by (Eq. (1)):

$$
\text { Percentage loading }=\frac{w_{1}-w_{0}}{w_{0}} * 100 \%
$$

Where $w_{0}$ is the initial mass $(\mathrm{g}) ; w_{l}$ is the mass after ZIF-8 in-situ grown (g)[9] .

\subsection{Adsorption experiments}

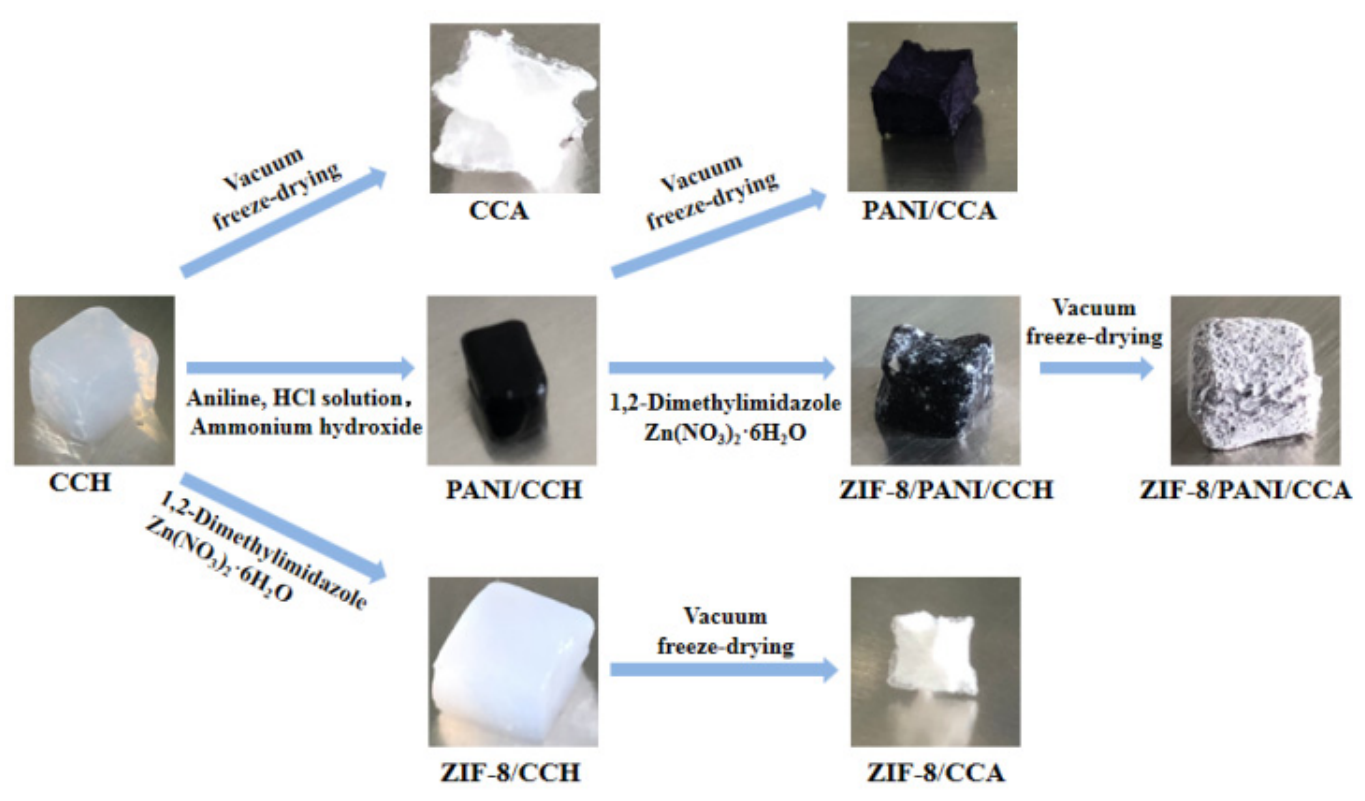

$0.01 \mathrm{~g}$ adsorbent was added into $20 \mathrm{~mL}$ solutions of TC $\left(c_{0}\right.$ of $\left.50 \mathrm{mg} \cdot \mathrm{L}^{-1}, \mathrm{pH}=7.0\right)$ and all the experiments were conducted under mechanical stirring with a speed of 150 $\mathrm{rpm}$ in darkness at $25^{\circ} \mathrm{C}$ for $6 \mathrm{~h}$. The concentration of TC solution was measured by UV-Visible spectrophotometry at $357 \mathrm{~nm}$.

The removal rate (\%) of tetracycline onto the adsorbent was listed as the following (Eq. (2):

$$
\text { Removal rate }=\frac{A_{0}-A_{1}}{A_{0}} * 100 \%
$$

Where $A_{0}$ is the absorbance of initial solution of TC; $A_{l}$ is the absorbance of residual liquid of TC[10].

\section{Results and discussion}

\subsection{Design of ZIF-8/PANI/CCA synthesis}

The synthesis process of ZIF-8/PANI/CCA is shown in

Fig. 1 .

Fig. 1. Synthetic route for ZIF-8/PANI/CCA and ZIF-8/CCA.

After vacuum freeze-dried, the coconut hydrogel $(\mathrm{CCH})$ turned into coconut aerogel (CCA). However, the structure of CCA has collapsed. PANI was coated onto $\mathrm{CCH}$ by in-situ polymerization of aniline, and the PANI/CCA was obtained after after vacuum freezedrying. The structure is well maintained, which indicating that PANI can increase the porosity of the material. After PANI/CCH was immersed in 2methylimidazole and $\mathrm{Zn}^{2+}$ solution, and ZIF8/PANI/CCH was obtained by in situ growth. After vacuum freeze-dried, a white and well structured ZIF8/PANI/CCA was obtained. After CCH was immersed in 2-methylimidazole and $\mathrm{Zn}^{2+}$ solution, and ZIF-8/CCH was obtained by in situ growth. The ZIF-8/CCA was obtained by vacuum freeze-drying. The morphology of ZIF-8/CCA is obviously better than that of CCA, indicating that the loading of zif- 8 is beneficial to the formation of the macro-structure of the material. The $\mathrm{H}$ atom of $\mathrm{O}-\mathrm{H}$ on $\mathrm{CCH}$ hydrogen bonds with the $\mathrm{N}$ atom on PANI, and the $\mathrm{H}$ atom of N-H on PANI also forms hydrogen bonds with the $\mathrm{O}$ atom on $\mathrm{CCH}$. The PANI layers were firmly bound on the surface of CCA and was assembled by this supramolecular. PANI as interface layers provid numerous quinone nitrogen atoms to coordinate with metal ions and generate rich nucleation sites favorably, which promote the in-situ growth of ZIF8 on CCA surface. The PANI not only ensures good mechanical adhesion but also promotes the formation of MOF nanoparticle arrays[8]. 


\subsection{Characterization of ZIF-8/PANI/CCA}

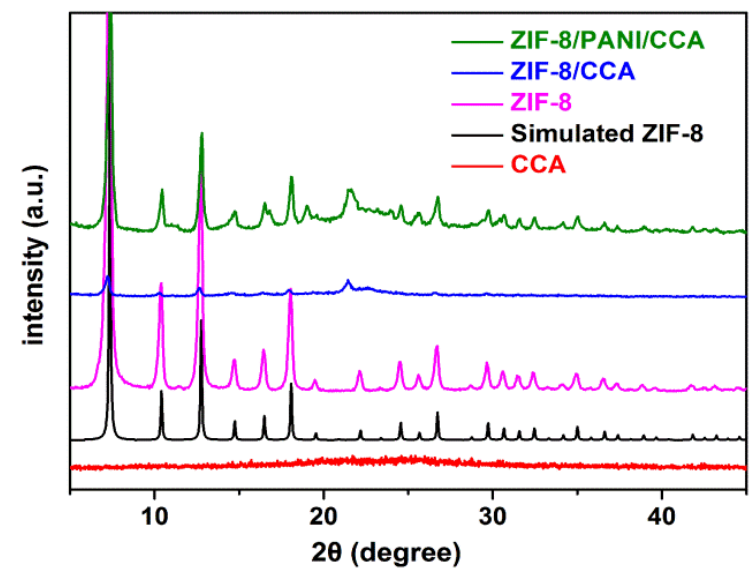

Fig. 2. The XRD of CCA, ZIF-8, ZIF-8/CCA and ZIF8/PANI/CCA.

In Fig. 2, the XRD curve of the synthesized pure ZIF-8 is consistent with the simulated ZIF-8 structure, indicating the successful synthesis process.The characteristic peaks of ZIF-8/PANI/CCA and ZIF8/CCA are consistent with pure ZIF-8, indicating that ZIF-8 has successfully grown on the surface of PANI/CCA and CCA. The peaks intensity of ZIF8/PANI/CCA is significantly higher than that of ZIF8/CCA, indicating that the loading rate of ZIF-8 on $\mathrm{PANI} / \mathrm{CCA}$ is significantly higher than that of CCA. PANI as interface layers can effectively promote the insitu growth of ZIF-8.

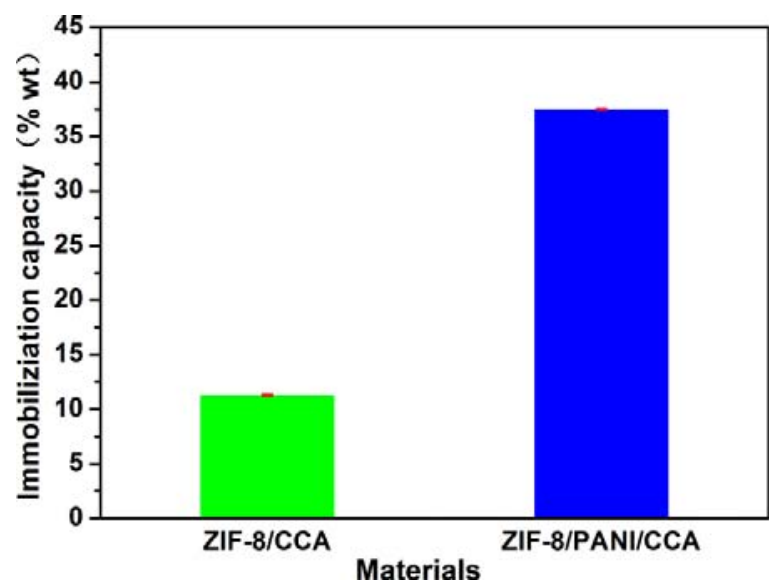

Fig. 3. Comparison of immobiliziation capacity of ZIF-8 on different arogel.

As shown in Fig. 3, the loading rates of ZIF-8 on CCA and PANI/CCA were $11.3 \%$ and $37.5 \%$, respectively, indicating that PANI can effectively promote the in-situ growth of ZIF-8 on coconut gel.

\subsection{Adsorption efficiency}

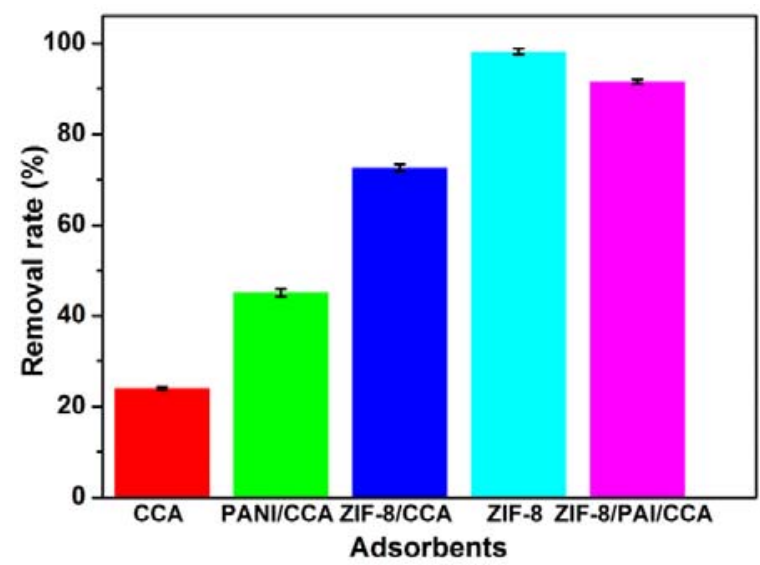

Fig. 4. Removal rate of TC by CCA, PANI/CCA, ZIF-8, ZIF-8/CCA and ZIF-68/PANI/CCA.

As shown in Fig. 4, ZIF-8/CCA has higher adsorption capacity toward tetracycline than CCA and PANI/CCA because of the ZIF-8 loading. ZIF-8/PANI/CCA has higher adsorption capacity toward tetracycline than ZIF8/CCA because of the ZIF-8 higher loading. But the adsorption capacity of ZIF-8/PANI/CCA is lower than that of ZIF-8 powders. This is because that the CCA and PANI/CCA have low adsorption capacity toward tetracycline. However, shaping powdery ZIF-8 into monolith can endow the adsorbents with good handleability and recycling properties that are more suitable for practical applications.

\section{Conclusion}

In conclusion, ZIF-8 was highly grown onto the surfaces of CCA with metal chelated layer PANI. XRD, was applied to characterize the structure of the aerogel, and the results confirmed that ZIF- 8 has successfully grown on PANI/CCA ZIF-8/PANI/CCA has higher adsorption capacity toward tetracycline than ZIF-8/CCA because of the ZIF-8 higher loading. Shaping powdery ZIF-8 into monolith can endow the adsorbents with good handleability and recycling properties that are more suitable for practical applications.

\section{References}

1. H.H. Yu, M.Y. Fan, Q. Liu, Z.M. Su, X. Li, Q.Q. Pan, X.L. Hu, Two Highly Water-Stable ImidazoleBased Ln-MOFs for SensingFe ${ }^{3+}, \mathrm{Cr}_{2} \mathrm{O}_{7}{ }^{2-} / \mathrm{CrO}_{4}{ }^{2-}$ in a Water Environment, Inorg. Chem. 59 (2020) 2005-2010.

2. C.H. Wang, J. Kim, J. Tang, J. Na, Y.M. Kang, M. Kim, H. Lim, Y. Bando, J.S. Li, Y. Yamauchi, Large-scale synthesis of MOF-derived superporous carbon aerogels with extraordinary adsorption capacity for organic solvents, Angew. Chem. 131 (2019) 1-6.

3. R. Zhao, T.T. Ma, S. Zhao, H.Z. Rong, Y.Y. Tian, G.S. Zhu, Uniform and stable immobilization of metal- 
organic frameworks intochitosan matrix for enhanced tetracycline removal from water, Chemical Engineering Journal 382 (2020) 122893.

4. C. Lei, J.K. Gao, W.J. Ren, Y.B. Xie, S.Y.H. Abdalkarim, S.L. Wang, Q.Q. Ni, J.M. Yao, Fabrication of metal-organic frameworks@cellulose aerogels composite materials for removal of heavy metal ions in water, Carbohydrate Polymers205(2019) 35-41.

5. X.T. Ma, Y. Lou, X.B. Chen, Z. Shi, Y. Xu, Multifunctional flexible composite aerogels constructed through in-situ growth of metal-organic framework nanoparticles on bacterial cellulose, Chemical Engineering Journal 356 (2019) 227-235.

6. Q. Liu, S.S. Li, H.H. Yu, F.M. Zeng, X. Li, Z.M. Su, Covalently crosslinked zirconium-based metalorganic framework aerogel monolith with ultralowdensity and highly efficient $\mathrm{Pb}(\mathrm{II})$ removal, Journal of Colloid and Interface Science 56 (2020) 1211219.

7. N. Xu, H.M. Gan, C. Qin, X.L. Wang, Z.M. Su, From Octahedral to Icosahedral Metal-Organic Polyhedra Assembled from Two Types of Polyoxovanadate Clusters, Angew. Chem. Int. Ed. 58 (2019) 46494653.

8. Q. Liu, H.H. Yu, F.M. Zeng, X. Li, J. Sun, X.L. Hu, Q.Q. Pan, C. Li, H. Lin, Z.M. Su, Polyaniline as interface layers promoting the in-situ growth of zeolite imidazole skeleton on regenerated cellulose aerogel for efficient removal of tetracycline. Journal of Colloid and Interface Science. 579 (2020) 119127.

9. H. Zhu, X. Yang, E. D. Cranston, S.P. Zhu, Flexible and porous nanocellulose aerogels with high loadings of Metal-Organic-Framework particles for separations applications, Adv. Mater. 28(2016) 7652-7657.

10.W.X. Yang, Y. Han, C.H. Li, L. Zhu, L.M. Shi, W.Z. Tang, J.L. Wang, T.L. Yue, Z.H. Li., Shapeable three-dimensional $\mathrm{CMC}$ aerogels decorated with $\mathrm{Ni} / \mathrm{Co}-\mathrm{MOF}$ forrapid and highly efficient tetracycline hydrochloride removal, Chemical Engineering Journal 375 (2019) 122076. 